\title{
A Review of Scientific Topics and Literature in Abdominal Radiology in Germany - Part 2: Abdominal Parenchymal Organs
}

\author{
Aktuelle Schwerpunkte und Literatur der Abdominalradiologie im \\ deutschsprachigen Raum - Teil 2: Parenchymatöse Oberbauchorgane
}

Authors

Affiliations
L. Grenacher ${ }^{1}$, M. S. Juchems² ${ }^{2}$ K. Holzapfel ${ }^{3}$, S. Kinner ${ }^{4}$, T. C. Lauenstein ${ }^{4}$, J. Wessling ${ }^{5}$, A. G. Schreyer ${ }^{6}$

Affiliation addresses are listed at the end of the article.

Key words
abdomen
pancreas
CT
liver
MRI

received $\quad 17.2 .2015$

accepted $\quad 6.7 .2015$

Bibliography

Dol http://dx.doi.org/

10.1055/s-0041-105411

Published online: 1.10.2015

Fortschr Röntgenstr 2016; 188:

245-252 @ Georg Thieme

Verlag KG Stuttgart · New York .

ISSN 1438-9029

\section{Correspondence}

Herr Prof. Andreas G. Schreyer Institut für Röntgendiagnostik, Universitätsklinikum

Regensburg

Franz-Josef-Strauß-Allee 11

93051 Regensburg

Germany

Tel.: ++ 49/0941/9447401

Fax: ++ 49/0941/9447402

andreas.schreyer@klinik.uni-

regensburg.de

\section{Zusammenfassung \\ $\nabla$}

Die AG Abdominal- und Gastrointestinaldiagnostik ist die Arbeitsgemeinschaft der Deutschen Röntgengesellschaft (DRG), die sich klinisch und wissenschaftlich auf Diagnostik und Therapie des Gastrointestinaltraktes mit allen parenchymatösen Abdominalorganen fokussiert. Im vorliegenden Artikel wird eine aktuelle Übersicht forschungsrelevanter Themengebiete vor allem der deutschsprachigen Radiologie gegeben. Die Experten der AG fassen dabei die aktuellen Studien bezüglich leberspezifischer Kontrastmittel zusammen. Ein besonderes Augenmerk wird auf die neue Einteilung der Leberadenomtypen mit ihren typischen Erscheinungsformen in der MRT gelegt. Ergänzt wird die Übersicht mit einer Zusammenfassung relevanter Publikationen zur radiologischen intraarteriellen Therapie von Lebertumoren mit Fokus auf der selektiven internen Radiotherapie (SIRT). Im Pankreas wird nach einer Übersicht der häufigsten Tumoren auf neue Entwicklungen der funktionellen Pankreasdiagnostik eingegangen. Abschließend werden die chronische Pankreatitis sowie die Autoimmunpankreatitis kurz im Spiegel der aktuellen Studienlage dargestellt.

Kernaussagen:

- Verschiedene Subtypen von Leberadenomen mit unterschiedlichen therapeutischen Konsequenzen können radiologisch MRT basiert differenziert werden.

- Extrazelluläres leberspezifisches Kontrastmittel sowie diffusionsgewichtete Bildgebung in der MRT der Leber sind stark befortschte radiologische Bildgebungsmethoden mit großem diagnostischen Potential.

- Bei Pankreasneoplasien sind funktionelle MRT Bildgebung mit Diffusion und Perfusion die aussichtsreichen neuen radiologischen Methoden.

\section{Abstract \\ $\nabla$}

The working group for abdominal and gastrointestinal diagnosis is a group of the German Radiological Society (DRG) focusing clinically and scientifically on the diagnosis and treatment of the gastrointestinal tract as well as the parenchymal abdominal organs. In this article we give an upto-date literature review of scientific radiological topics especially covered by German radiologists. The working group experts cover the most recent relevant studies concerning liver-specific contrast media with an emphasis on a new classification system for liver adenomas. Additionally studies regarding selective internal radiotherapy (SIRT) are reviewed. For the pancreas the most important tumors are described followed by an introduction to the most recently introduced functional imaging techniques. The manuscript concludes with some remarks on recent studies and concerning chronic pancreatitis as well as autoimmune pancreatitis.

Key Points:

- Different subtypes of liver adenomas with different therapeutic consequences can be differentiated by MRI

- Most recently published studies focus on liver imaging with extracelluar liver specific contrast media as well as diffusion weighted imaging. They consider this new method having a high diagnostic potential.

- For pancreatic neoplasm diagnosis diffusion as well as perfusion - imaging is considered as a highly promising method.

Citation Format:

- Grenacher L, Juchems MS, Holzapfel K et al. A Review of Scientific Topics and Literature in Abdominal Radiology in Germany - Part 2: Abdominal Parenchymal Organs. Fortschr Röntgenstr 2016; 188: 245-252 


\section{Introduction}

\section{$\nabla$}

In part one of our literature review regarding the gastrointestinal tract we already made the expertise of the members of the working group for abdominal and gastrointestinal diagnosis of the German Radiological Society available to all members of the DRG. The working group is a representative of German abdominal radiology and a comprehensive platform for interested researchers and clinicians with a focus on abdominal diagnosis and therapy. This second part includes an overview of the current literature with a focus on the developments and trends related to the abdominal parenchymal organs such as the liver and the pancreas.

The use of liver-specific contrast agents with uptake in the hepatocytes via an organic anion-transporting polypeptide (OATP) has resulted in a significant improvement in differential diagnosis. After the original euphoria regarding the exact differentiation of the hepatocarcinogenesis, a certain amount of sobering has occurred primarily as a result of several Japanese studies. A liverspecific contrast agent seems to be helpful for the subtyping of liver cell adenomas and their differentiation from FNH (focal nodular hyperplasia). The current status for the subtyping of liver cell adenomas is therefore briefly discussed in this overview. In addition, the value of SIRT (selective internal radiotherapy) is presented on the basis of the current literature.

The second part of this overview addresses the pancreas. In addition to the high mortality rate compared to other tumor entities, the pancreas has increasingly become a topic of interest in research and at conferences due to the predicted dramatic increase in the number of cases of cancer for the next 20 years. This article presents the standards and the value of new techniques with functional imaging. Finally, a summary of the current results of the S3 guidelines conference regarding chronic pancreatitis and autoimmune pancreatitis is briefly presented.

\section{Liver}

$\nabla$

The scientific studies regarding imaging of the liver published in recent years in the literature in Germany are largely from members of the working group for abdominal and gastrointestinal diagnosis or were conducted with their active participation.

The value of the liver-specific contrast agent Gd-EOB-DTPA (Primovist) regarding the diagnosis of focal liver lesions was further examined [1-4]. A large study proved, that the uptake of GdEOB-DTPA in the hepatobiliary contrast phase is the best criterion in the differentiation between $\mathrm{FNH}$ and heptocellular adenoma and this criterion is superior to all morphological and dynamic criteria in the differentiation of the two lesions [1]. The nomenclature of adenomatous lesions was constantly changing in the past. In 2010a new standard was presented by a French working group in a newly published classification system [5]. There are currently 3 adenoma subtypes with varying frequencies (see parentheses) and an unclassified subgroup to which various tendencies for malignant change and thus divergent treatment approaches can be assigned:

- HCA with HNF1 $\alpha$ mutation: No tendency for malignant change $(35 \%)$

- HCA with $\beta$-catenin mutation: High tendency for malignant change (10\%)

- Inflammatory HCA (IHCA) (45\%)

- Unclassified HCA (10\%)

In a recent study presented at ESGAR 2014 including a total of 39 systematically analyzed and histologically verified liver cell adenomas, characteristics for the subtyping of liver cell adenomas using Gd-EOB-DTPA were developed [6]: All adenomas showed a hypointense signal behavior in the hepatobiliary phase after 10 min p.i. in contrast to the regular hyperintensity of FNH in this phase. Under consideration of the qualitative and quantitative results of this study, the unenhanced T1 sequence and the venous contrast phase seem to be diagnostically valid for the differentiation between inflammatory HCA and HNF1 $\alpha$ adenoma
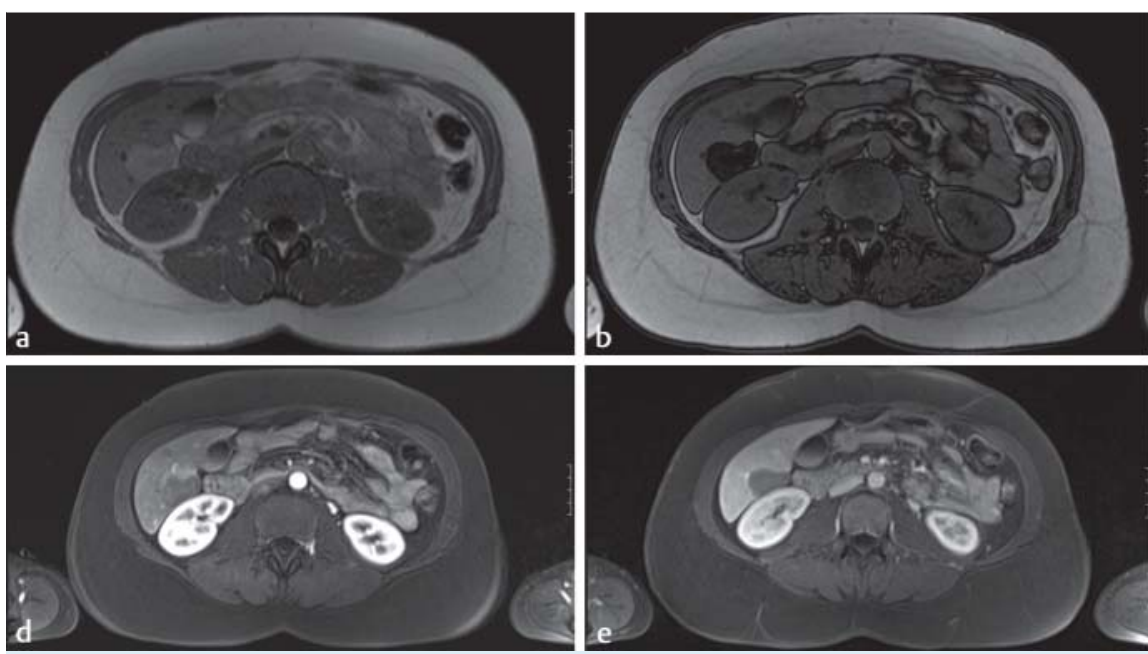

Fig. 138 -year-old asymptomatic patient with histologically verified $\mathrm{HNF} 1 \alpha$ adenoma in seg. VI: a T1 fl2 d tra In-phase; b T1 fl2 d tra Opposed-phase; c T2 HASTE tra; $\mathbf{d}$ T1 $\mathrm{fl} 3 \mathrm{~d}$ tra contrast agent arterial phase; e T1 fl3 d tra contrast agent venous phase; $\mathbf{f} \mathrm{T} 1 \mathrm{fl} 3 \mathrm{~d}$ tra contrast agent hepatobiliary phase after $10 \mathrm{~min}$. Typical fat detection of HNF1 $\alpha$ adenoma in the Opposedphase $\mathbf{b}$, no significant hyperarterialization $\mathbf{d}$, hypointensity already in the venous phase $\mathbf{e}$ and typical hypointensity in the hepatobiliary phase $\mathbf{f}$.
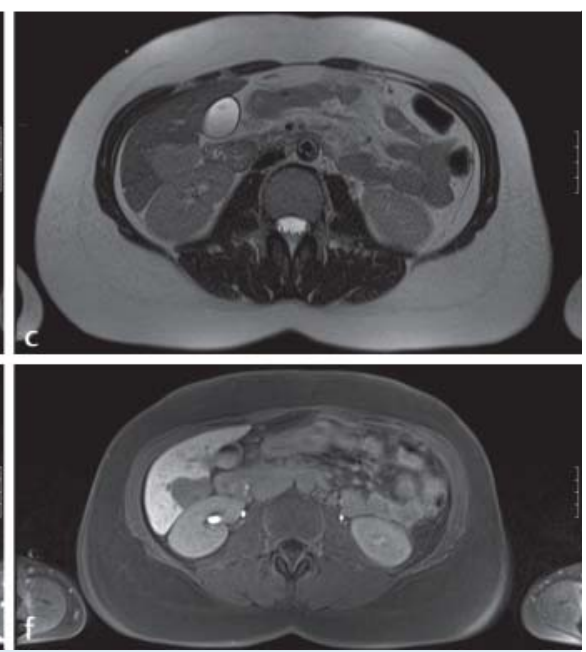

Abb. 1 38-jährige asymptomatische Patientin mit histologisch gesichertem HNF1 $\alpha$ Adenom in Seg. VI: a T1 fl2 d tra In-phase; b T1 fl2 d tra Opposed-phase; c T2 HASTE tra; $\mathbf{d}$ T1 fl3 d tra KM arterielle Phase; e T1 fl3 d tra KM venöse Phase; $f$ T1 fl3 d tra KM hepatobiliäre Phase nach 10 Min. Typischer Fettnachweis des HNF1 $\alpha$ Adenoms in der Opposed-phase $\mathbf{b}$, keine deutliche Hyperarterialisierung $\mathbf{d}$, bereits Hypointensität in der venösen Phase e sowie typischerweise Hypointensität in der hepatobiliären Phase f. 
( $\bullet$ Fig. 1). Primarily iso- to hyperintense signal behavior of inflammatory adenomas in the unenhanced T1 fs sequence and isointensity persisting into the venous phase are typical. Due to their typical fat content, HNF1 $\alpha$ adenomas appear hypointense in the fat-saturated $\mathrm{T} 1$ sequence and show venous washout and hypointensity already $50-60$ s post-injection. A significant point seen only in the qualitative evaluation is the hyperarterialization of the IHCAs compared to HNF1 $\alpha$ adenomas that tend to appear isointense. The very rare subtype of $\beta$-catenin-mutated adenomas was not seen in this collective so that no statement about any typical MR morphologies can be made. On the whole, MRI using the liver-specific contrast agent GD-EOB-DTPA is a reliable method for identifying inflammatory hepatocellular adenoma (IHCA) versus HNF1 $\alpha$ adenoma.

In addition, it was shown in a multicenter study that Gd-EOBDTPA-enhanced MRI is superior to both CT and MRI using extracellular Gd-chelate as the contrast agent in the diagnosis of liver metastases of colorectal cancer [4]. Moreover, a role of Gd-EOBDTPA-enhanced imaging of bile ducts in the preoperative evaluation of live liver donors was published [7].

The role of diffusion-weighted MRI (DWI) in liver diagnosis was also examined. Among other things, DWI was compared to GdEOB-DTPA-enhanced MRI [8]. It was shown that similarly good results regarding the detection of focal liver lesions can be achieved with DWI as with a liver-specific contrast agent. The combination of DWI and Gd-EOB-DTPA-enhanced MRI is significantly superior to the use of either modality alone (i.e., only DWI or only Gd-EOB-DTPA). Moreover, it was thus shown that changes caused by MR-guided radiofrequency ablation of HCCs or metastases can be visualized via DWI already during or shortly after the intervention [9].

Numerous studies conducted last year examine the value of PET/ MRI in oncological imaging. Although an examination of the entire body is usually performed, the use of MR components is also important for the detection and characterization of focal liver lesions. Therefore, PET/MRI was significantly superior to PET/CT in a study regarding this topic with respect to both points [10].

However, despite all technical developments, the experience of the radiologist is an important parameter. This was demonstrated in a study that examined the influence of the radiologist's experience on the diagnostic accuracy in the detection of liver metastases in patients with colorectal tumors [11]. In comparison to CT examinations, the influence of the radiologist's experience was even more pronounced in the case of MRI examinations.

A further interesting development regarding systematic radiological evaluation of liver masses in recent years is the Li-RADS (Liver Imaging Reporting and Data System) published by the ACR (American College of Radiology) in 2011 [12]. With standardized terminology and a classification system for CT and MRI, the system allows structured evaluation of individual liver lesions (referred to as observation) for patients with liver cirrhosis or other risk factors for hepatocellular carcinomas. Basically, individual liver masses are classified in a defined 5-category system (definitely benign, probably benign, intermediate, probably HCC, definitely HCC) on the basis of 5 major features, such as increase in size or typical contrast behavior. The system is still being further developed and was last modified in 2014. Additional revisions can be expected. As a result of the lack of evaluation regarding outcome analyses and the lack of prospective studies of the system, it is viewed critically yet optimistically.

\section{Intra-arterial treatment of liver tumors}

$\nabla$

Primary and secondary liver tumors are often part of diagnostic as well as therapeutic radiology in the clinical routine. Depending on the extent of the tumor invasion, different treatment concepts can be used. In addition to surgical and medication-based treatments, minimally invasive local or locoregional methods, such as radiofrequency ablation (RFA) or transarterial chemoembolization (TACE), are often used. A newer form of endovascular treatment is selective internal radiotherapy (SIRT). This is a transarterial, catheter-based method in which the beta-emitter yttrium-90 is introduced into the liver. The radionuclide is bound to glass or resin microspheres. As a result of increased accumulation in particular in hypervascularized liver tumors, a focused radiotherapeutic effect is achieved.

Although SIRT is similar to TACE in many respects, precautionary safety measures must be taken to avoid a transfer of yttrium to extrahepatic tissue. In recent years the handling of "critical" vessels has been a topic of research. Such critical vessels include for example the gastroduodenal artery, the cystic artery, and the falciform artery, which usually arise from the vascular bed of the hepatic artery and provide the arterial supply to the GI tract, the gallbladder and the abdominal wall, respectively. For example, the gastroduodenal artery is routinely closed prior to SIRT in many centers. In a study by Schelhorn et al. [13], 86 patients in whom coil embolization of the gastroduodenal artery was performed were followed up. It was able to be shown that new collaterals into the flow region of the gastroduodenal artery that had to be closed prior to yttrium administration formed in 28 of 86 patients after vascular occlusion. However, these collaterals could not be probed and occluded in 3 patients so that these patients could not undergo SIRT. The authors concluded that the gastroduodenal artery should not be occluded and instead segmental or lobar SIRT should be performed with the microspheres being applied distal to the origin of the gastroduodenal artery.

Another vessel that is a focus of SIRT is the cystic artery. If yttrium particles spread to the gallbladder, radiation-induced cholecystitis can occur. Theysohn et al. [14] reported on a series of 20 patients in whom special measures were necessary prior to yttrium therapy in order to prevent or reduce the spread of yttrium to the gallbladder wall. The cystic artery was occluded temporarily with gel particles or permanently with coils. Ischemia of the gallbladder wall did not occur in the patients. However, it was able to be ensured by these measures that no microspheres entered the gallbladder bed.

The falciform artery is categorized as problematic less frequently. Since the falciform artery often originates far in the periphery of the hepatic artery, selective occlusion is often not possible. Schelhorn et al. examined 19 SIRT patients with an open falciform artery [15]. It was able to be shown that a large-caliber, open falciform artery is not a contraindication for SIRT. The authors recommended placing cold packs on the abdominal wall during yttrium administration in cases in which the falciform artery cannot be occluded. This reduces the arterial blood flow into this area and the perfusion with yttrium is minimized.

In addition to the rather technical studies mentioned above, a further focus of scientific interest is the question as to whether it is useful to combine SIRT with other therapeutic methods. A multicenter study (SIRFLOX study) examines patients with liver metastases in colorectal cancer [16]. The extent to which SIRT in addition to chemotherapy with FOLFOX extends the progressionfree survival of patients is examined. The SORAMIC study has a 
similar study design [17]. The effect of a combination of SIRT and sorafenib in HCC patients is evaluated. Initial results show good tolerability of the treatment combination. However, the therapeutic benefit must first be proven in larger patient collectives. Under clinical conditions, a positive effect of sorafenib prior to SIRT could already be described [18]. Theysohn et al. examined patients in whom SIRT was initially not possible due to a high hepatopulmonary shunt fraction. Sorafenib therapy resulted in a significant reduction of the pulmonary shunt so that SIRT could consequently be performed in select patients.

\section{Pancreas}

$\nabla$

\section{Solid pancreatic tumors - ductal adenocarcinoma}

The most common solid tumor of the pancreas is the ductal adenocarcinoma that comprises approximately $95 \%$ of tumors of the exocrine pancreas. Approximately 15000 patients are diagnosed each year in Germany with this tumor and the annual mortality rate for this entity is similarly high. Despite the advances in diagnosis and treatment, the prognosis remains unfavorable. 80-90\% of patients have an inoperable tumor at the time of diagnosis.

The recommendation of the S3 guidelines updated in 2013 regarding imaging methods for primary diagnosis is: "The firstchoice diagnostic methods for detecting pancreatic cancer are upper abdominal ultrasound, endoscopic ultrasound, MD-CT examination, and MRI in combination with MRCP" [19]. A definitive recommendation for or against one of the methods is not made. The method with which the examiner has the greatest experience should be used.

Imaging in adenocarinoma of the pancreas has 3 main goals:

- Detection or exclusion of a tumor

- Evaluation of the resectability of a carcinoma

- Differentiation of ductal adenocarcinoma from other tumor types or from chronic pancreatitis

In the case of early diagnosis, tumor resection seems possible. This means a possible improvement of the survival rate for a select group of patients $[20,21]$.

Since these pancreatic tumors have a ductal origin, an obstruction and duct dilatation are the earliest signs of a tumor. Tumors with a size of less than $1 \mathrm{~cm}$ can be detected solely on the basis of duct dilatation in imaging in approximately $60 \%$ of cases [22]. Approximately $70 \%$ of ductal adenocarcinomas are located in the head of the pancreas which is why obstruction of the distal bile duct (double duct sign) occurs relatively frequently and early [23].

After contrast administration, pancreatic cancer is typically less perfused than the surrounding tissue on CT and MRI. Around the tumor the pancreas loses the typical lobulated contour. In addition, tumors show direct infiltration into the surrounding retroperitoneal fatty tissue very early. Pancreatic cancer spreads quickly lymphatically and perineurally into the retroperitoneal fatty tissue and grows around the mesenteric vessels and the celiac trunk.

After the diagnosis of pancreatic cancer, the assessment of resectability or irresectability is the greatest challenge for imaging. Irresectability can be determined more reliably. The S3 guidelines specify the following recommendation regarding preoperative diagnosis: "MD-CT and endoscopic ultrasound are recommended for the preoperative evaluation of local tumor extension and for evaluating resectability. Abdominal ultrasound is mandatory for evaluating systemic tumor spread. Abdominal MD-CT is mandatory when systemic formation of metastases cannot be detected

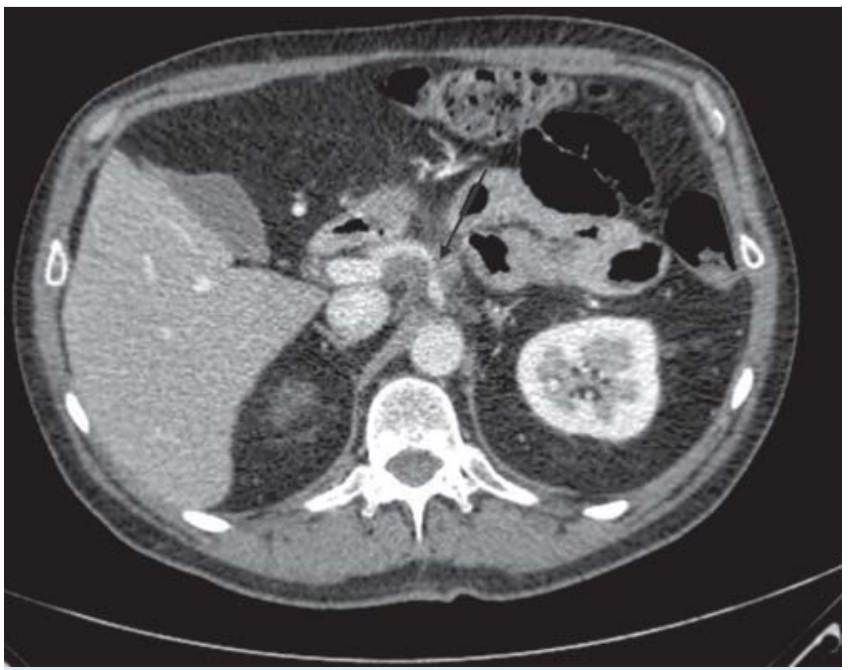

Fig. 2 Signs of irresectability in a ductal adenocarcinoma of the pancreas: Surrounding of the celiac trunk by more than $180^{\circ}$ and narrowing of the vessel (arrow).

Abb.2 Zeichen der Irresektabilität bei einem duktalen Adenokarzinom des Pankreas: Deutliche Ummauerung des Tr. coeliacus um mehr als $180^{\circ}$ und Einengung („narrowing“) des Gefäßes (Pfeil).

on abdominal ultrasound or when imaging according to RECIST criteria ("response evaluation criteria in solid tumors") is desired in study situations [19].

The following irresectability criteria are defined:

- Extensive infiltration of the celiac trunk and/or of the superior mesenteric artery

- Distant metastases

While every vascular contact of the tumor was previously evaluated as a sign of infiltration, surrounding of the superior mesenteric artery or the celiac trunk of more than $180^{\circ}$ is now considered a reliable sign of irresectability ( $\bullet$ Fig. 2) [24].

\section{Endocrine pancreatic tumors}

Rare endocrine pancreatic tumors include a heterogeneous group of diseases with different clinical pictures, different morphologies, and varying degrees of malignancy. The diagnosis of these tumors is typically based on the combination of characteristic clinical symptoms and lab findings, particularly in the case of hormonally active tumors. The goal of imaging is to locate tumors and detect metastases. The two most common tumors, insulinoma and gastrinoma, have an annual incidence of $0.3-3$ per million people while the remaining tumor types occur much less frequently.

\section{Cystic pancreatic tumors}

Cystic pancreatic tumors are discovered using high-resolution imaging methods with increasing frequency and are responsible for the increase in surgical interventions involving the pancreas. The following tumors represent approximately $90 \%$ of pancreatic lesions with a cystic appearance $[25,26]$ :

- Intraductal papillary mucinous neoplasms (IPMN)

- Mucinous cystic neoplasms (previously referred to as mucinous cystadenomas)

- Serous cystic neoplasms (previously referred to as serous cystadenomas)

- Solid pseudopapillary tumors (Frantz's tumors)

- Neuroendocrine cystic tumors 
Cystic pancreatic tumors typically have a series of clinical and morphological features that allow characterization in most cases.

\section{Intraductal papillary mucinous neoplasms (IPMN)}

Histologically speaking, an IPMN is based on the intraductal epithelial proliferation of cells that are polyp-like or flat and are usually associated with an excessive formation of mucus. The excessive viscous mucus displaces the pancreatic ducts and causes them to dilate thus giving these tumors their cystic appearance. Strictly speaking, this is not a "cystic" tumor of the pancreas but only cystic dilatation of the pancreatic duct system. A communicating system of cystically dilated ducts that look like cysts on cross-sectional images but differ clearly from the grape or honeycomb-like appearance of serous cystic neoplasms typically form. The papillary tumors can seem like small polyps and can be identified starting at a size of approximately $3 \mathrm{~mm}$, particularly after contrast administration.

IPMNs are categorized according to their location and type of growth as main-duct types, side-branch types, and mixed types. The mixed types seem to be neoplasms that arise from the main duct and spread secondarily into the side branches [27]. The sidebranch type is usually located in the head of the pancreas and preferably in the uncinate process. A side-branch IPMN histologically corresponds to a gastric type while the main-duct IPMN is differentiated as a pancreato-biliary or intestinal type. The main-duct type has a significantly higher tendency for malignant change than the side-branch type. A differentiation is made between benign tumors, borderline types, and malignant tumors. A cancer in situ is found in up to $27 \%$ of cases and an invasive cancer in up to $40 \%$ of cases. Histologically speaking, invasive types are either mucinous or ductal carcinomas. The 5-year survival rate is $98-100 \%$ for noninvasive tumor types, $89 \%$ for minimally invasive cancers, and $58 \%$ for invasive cancers [28].

Depending on the type of spread, the following changes are characteristic on CT and MRI:

- Cystic dilatation of the side branches

- Circumscribed or diffuse dilatation of the main duct

- Small intraductal papillary tumors

In the case of the side-branch type, the detection of communication with the pancreatic duct system is essential for the delimitation from other tumors ( $\mathrm{Fig} .3$ ). The introduction of thin-slice CT made it possible to show this connection in over $80 \%$ of cases. However, reconstruction methods must be used. This detection is possible in approximately $90 \%$ of cases with MRCP.

In the case of the main-duct type, segmental or diffuse dilatation of the pancreatic duct is pathognomic and is frequently associated with polypous intraductal changes ( $\bullet$ Fig.4).

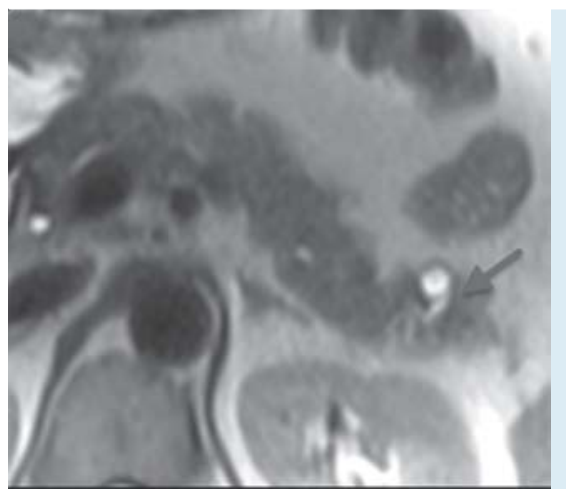

Fig. 3 Small sidebranch IPMN: Clear communication of the cystically dilated side duct with the main duct (arrow).

Abb. 3 Kleine Seitengang-IPMN: Eindeutige Kommunikation des zystisch erweiterten Seitengangs mit dem Hauptgang (Pfeil).
In principle, it is possible to differentiate between benign and malignant types of IPMN [29]. Main-duct IPMNs must be fundamentally classified as potentially malignant. Patients with dilatation of the main duct and a corresponding physical condition should undergo surgery. In contrast, patients with side-branch IPMN without high-risk stigmata (obstructive jaundice in patients with a cystic lesion in the head of the pancreas, solid portion and/or diameter $>10 \mathrm{~mm}$ ) can be monitored [30].

\section{Mucinous cystic neoplasm}

According to the new WHO classification, mucinous cystic neoplasms are characterized as follows: "Tumors without a connection to the pancreatic duct system that have a muciferous epithelium and often have an ovarian-like stroma" [31]. The histological detection of an ovarian-like stroma is because this tumor type is found almost exclusively in women. The age of onset is usually between the 40th and 50th year of life. Mucinous cystic tumors are typically solitary and are comprised of individual or a few large, thick-walled cysts that have a chambered appearance in some cases. Almost all tumors (>95\%) are in the corpus and cauda of the pancreas. The cysts contain mucinous, hemorrhagic or necrotic material. Nodular changes in the wall and papillary projections can be indications of a malignant change [32].

Imaging is dependent on the tumor architecture and cyst contents. In particular, the detection of a hemorrhage is a strong indicator of this type of tumor. High signal intensity caused by bleeding and protein-rich mucus is seen on T1-weighted MRI sequences. The cyst contents are signal-intense on T2-weighted images and even thin cyst walls are sharply defined in the fluid. Cyst walls and septae visibly absorb contrast agent. Although calcifications can be better detected with CT, septations usually cannot be delimited as well as with MRI.

\section{Serous cystic neoplasm}

Serous cystic neoplasms include the following tumors:

- Serous microcystic adenoma

- Serous oligocystic adenoma

- Cystic neoplasms in Von Hippel-Lindau syndrome

- Rare serous cystadenocarcinoma

The most common subtype is microcystic adenoma comprised of numerous small cysts with a diameter of $1-5 \mathrm{~mm}$. A central scarred connective tissue structure from which radial septae arise and that can contain calcifications is common. Serous oligocystic adenomas are slightly rarer and comprise approximately $7-10 \%$ of all serous cystic tumors. They are comprised of individual or a few larger cysts with a size of $2-25 \mathrm{~mm}$. They have no central scar and no calcifications but have the same histological signs as serous microcystic adenomas. Cystic neoplasms in Von HippelLindau syndrome are usually numerous and are distributed in a diffuse or multifocal manner over the entire pancreas [33].

The morphological visualization of these tumors depends on their architecture, e.g. the size of individual cysts, and the spatial resolution of the technique being used. If the cysts are very small and the septae are delicate, they can only be sufficiently visualized with very high resolution. In the case of insufficient resolution, these tumors can give the appearance of a solid aspect.

\section{Solid pseudopapillary pancreatic tumor}

This tumor, also referred to as "Frantz's tumor" occurs almost exclusively in young women (average age 24 - 39 years) and is seen in children in individual cases. However, this mass is typically classified as benign or with low malignant potential. The prog- 
nosis is favorable [34]. If these tumors are seen in older patients, the probability of a malignant change increases. The most common metastatic pathway in the case of a malignant change is hematogenic spread into the liver. Tumors can be well delimited on MRI and CT and have a very varied appearance depending on their architecture. Small tumors (diameter of $<3 \mathrm{~cm}$ ) are often solid, can be well delimited and show slow contrast enhancement after intravenous contrast administration [35, 36]. Only larger tumors show the characteristic presentation with solid and cystic areas with hemorrhagic areas standing out in MRI in particular. A thick capsule that shows contrast enhancement can typically be detected. Peripheral calcifications are described in up to $30 \%$ of tumors. This tumor is often misinterpreted as a (post-) traumatic pseudocyst.

New trends in pancreas diagnosis - functional imaging In the special research area SFB/TR125 "cognition-guided surgery", the Heidelberg working group examined new imaging methods such as dual-energy CT perfusion (known as DECT perfusion) and diffusion MRI (DWI-MRI) regarding their value for pancreas diagnosis in two SFB projects.

Using DECT perfusion on color-coded perfusion maps typical hypodense pancreatic tumors can be reliably identified and small isodense tumors can appear as focal hypoperfused areas that can be determined quantitatively as well as qualitatively [37, 38]. In addition to the two-compartment model (Patlak model) for perfusion map calculation, it is important to also use motion correction algorithms [39]. The value of DECT perfusion for the identification of pancreatic tumor relapse in early postoperative follow-up is the topic of current research.

Diffusion MRI is becoming increasingly important in oncology and thus in abdominal radiology. In addition to the liver, diffusion sequences are increasingly used for pancreas diagnosis. However, the sequence technique from the liver cannot be transferred on a 1:1 basis due to the different microcirculation structures of the vessels in the pancreas.

Basically, two different models are used for diffusion imaging of the pancreas: the monoexponential method and the biexponential method.

The former method is faster and more widely available and typically uses two $b$-values for tumor detection $(b=0$ and 1000) and four b-values for lesion detection $(0,100,500,1000)$ [40].

The resulting ADC maps correspond to the diffusion effect in the analyzed voxel. The less known and more time-intensive biexponential method combines tissue perfusion with the diffusion component, microcapillary perfusion being visualized at low b-values in particular. Low b-values of less than $100 \mathrm{sec} / \mathrm{mm} 2$ are more sensitive for perfusion and microcirculation effects. However, measurements of the up to $11 \mathrm{~b}$-values needed for the acquisition in addition to the longer acquisition time are significantly more complex and also require more complex post-processing. However, a differentiation between pancreatitis, carcinoma and fibrosis is possible when there is a certain overlap of both collectives [41]. Therefore, this method is currently not used in the clinical routine and is only evaluated in studies. At $1.5 \mathrm{~T}$, monoexponential models as mentioned above are therefore currently recommended even if the authors recommend consideration of the biexponential method for diffusion imaging of the pancreas based on data from larger study collectives [42]. The value of DWI-MRI for the follow-up of autoimmune pancreatitis is currently being examined.

\section{Chronic pancreatitis}

After a long preparation period, the first German S3 guidelines regarding the diagnosis and treatment of chronic pancreatitis were developed and published in 2012 under the supervision of the German Society of Digestive and Metabolic Diseases with participation of the German Radiological Society [43]. Basically, the guideline recommendes the use of ultrasound for basic diagnosis in this complex and increasingly prevalent chronic disease [44]. According to current literature, MRI in combination with MRCP is the optimal method for differentiating pancreatic cancer from focal changes in chronic pancreatitis. With respect to study design, categorization according to the modified Cambridge classification is recommended [45].

\section{Autoimmune pancreatitis (AIP)}

Autoimmune pancreatitis is a rare special form of idiopathic chronic pancreatitis with pain, cholestatic jaundice, and hypergammaglobulinemia of all classes primarily of IgG4, described for the first time in 1995 [46]. Since the increase in immunoglobulins results in systemic inflammatory fibrosis, the pancreas is only an effector organ. Autoimmune pancreatitis responds well to cortisone and should therefore be definitively radiologically differentiated from the most important differential diagnosis, i. e., pancreatic cancer which requires a completely different treatment [43].

In addition to histological and serum changes for reliable diagnosis, there are some typical signs that can be effectively detected with radiology [47]:

a) The "sausage sign", a generalized sausage-shaped thickening of the entire pancreas without significant peripancreatic fluid quantities that can be diagnosed equally with CT and MRI. Differentiation from acute edematous pancreatitis is not possible here.

b) An area that is hypointense on T1-weighted images compared to the rest of the parenchyma and is slightly hyperintense on T2-weighted images can be delimited in unenhanced MRI sequences in approximately $50 \%$ of cases of AIP. Hypointensity is seen in the arterial phase of contrast dynamics and a hyperintense signal known as "late enhancement" is seen in approx. $65 \%$ of cases in the venous phase and in the late venous phase in $74 \%$ of cases. This hyperintense signal in the venous and late venous phase indicates an AIP. Such a hyperdense behavior in the venous phase often cannot be delimited with CT. Therefore, MRI is superior to $\mathrm{CT}$ for the diagnosis of AIP.

Initial studies regarding the diagnosis and treatment course of AIP via DWI-MRI are the current topic of research.

Cave: the two clinical types of AIP, lymphoplasmacytic sclerosing type 1 (LPSP) and idiopathic duct-centered type 2 (IDCP), cannot be radiologically differentiated from one another.

\section{Developments, summary and conclusion}

Concerning the current US cancer statistics, a dramatic change in the incidence and mortality in one of the fastest growing medical areas, namely oncology, is forecast by the year 2030 .

In addition to a doubling of the number of cases in oncology in the next 30 years, the number of cases of pancreatic cancer and hepato-biliary tumors in particular will increase significantly while the number of cases of colorectal tumors will slightly decrease and the number of cases of bronchial cancer and breast cancer will increase only slightly [48]. 

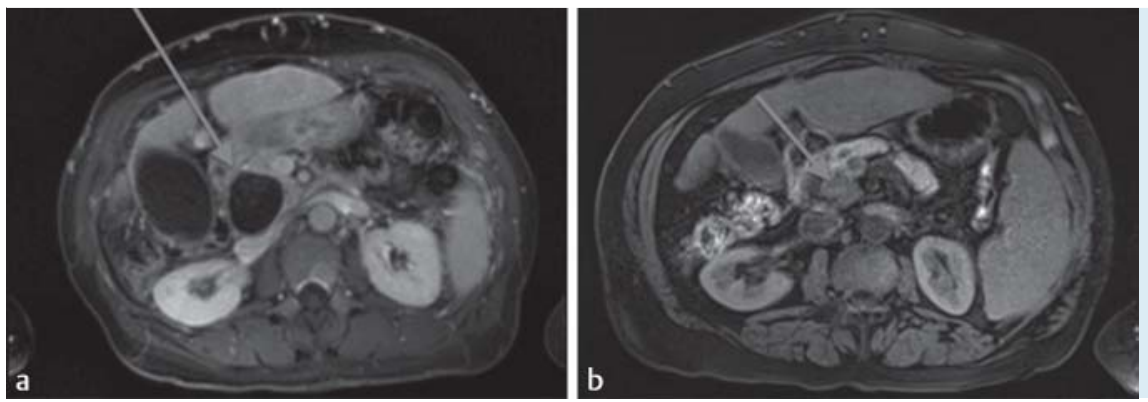

Fig. 4 Malignant change of a main-duct IPMN a detection of a very large main-duct IPMN with purely cystic dilatation (arrow). b The same patient 3 years later: Clear, solid tumor portions (arrow) are signs of a malignant change.

Abb. 4 Maligne Entartung einer Hauptgang IPMN a Nachweis einer sehr großen Hauptgang IPMN mit rein zystischer Erweiterung (Pfeil). b Derselbe Patient 3 Jahre später: Es finden sich nun deutliche, solide Tumoranteile (Pfeil) als Zeichen einer malignen Entartung.

This has major effects on radiological diagnosis and interventional therapy, in particular in abdominal radiology. Publications regarding the use of liver-specific contrast agents for differentiating the hepatocarcinogenesis and the evaluation of the treatment success of new techniques such as SIRT are both of great interest because with an increasing number of oncological patients the number of treatments in general as well as the number of patients requiring palliative treatment will increase significantly and all involved disciplines will encounter capacity limits. Primarily systematic studies on SIRT therapy allowed optimization of the procedure. In addition to exact diagnosis determination, radiology could play an important role in an increasingly personalized medicine, namely for answering the following questions: which patient is suitable for which treatment, is this specific (and usually very expensive) treatment effective, and should the treatment be continued?

Therefore, the countless scientific activities in German literature and the continuous increase in the number of members in the working group for abdominal radiology of the German Radiological Society can be sufficiently explained.

The known paradigm changes in almost all new S3 guidelines with an increasing influence of cross-sectional imaging methods and interventional treatments are based on the good amount available data due to the high number of publications particularly in the German literature. In addition to the evaluation of whether a patient with newly diagnosed pancreatic cancer is suitable for a surgical treatment, cross-sectional imaging with the highest spatial resolution should also be used for the early diagnosis of a local relapse without distant metastases because repeat surgery yields a survival advantage of approximately 11 months [49]. The same is true for the constantly increasing incidental diagnosis of cystic pancreatic lesions, the correct diagnosis of type, the typing of a possible IPMN and the evaluation of the presence of a possible malignancy with a corresponding therapy procedure. Whether the increasing number of publications regarding functional oncological imaging will become established in the clinical routine is clarified by current publications regarding the differentiation of hypodense and isodense pancreatic tumors via DECT perfusion with color-coded perfusion maps regarding blood flow, blood volume, and permeability in the framework of German Research Foundation-supported special research areas. Whether a differentiation between pancreatitis and carcinoma via DWI-MRI is possible and what the value of diffusion MRI will be in the future are also topics of current research [32-35].

In summary, radiologists have become an established part of modern oncology in an interdisciplinary setting and today are much more than "image demonstrators" and will allow these regions to grow significantly according to the predicted increase in hepato-biliary tumors and pancreatic cancer with a special focus on abdominal radiologists and interventional colleagues by 2030 .

\section{Affiliations}

Diagnostik München - Diagnostic Imaging Center, Munich, Germany

Diagnostic and Interventional Radiology, Konstanz Hospital, Konstanz, Germany

Technische Universität Munich, Department of Radiology, Munich, Germany

4 Department of Radiology, University Hospital Essen, Germany

Department of Radiology, Clemens Hospital, Muenster, Germany

Department of Radiology, University Hospital Regensburg, Germany

\section{References}

1 Grieser C, Steffen IG, Kramme IB et al. Gadoxetic acid enhanced MRI for differentiation of FNH and HCA: a single centre experience. Eur Radiol 2014; $24: 1339-1348$

2 Haimerl M, Wachtler M, Platzek I et al. Added value of Gd-EOB-DTPAenhanced Hepatobiliary phase MR imaging in evaluation of focal solid hepatic lesions. BMC medical imaging 2013; 13: 41

3 Haimerl M, Wachtler $M$, Zeman $F$ et al. Quantitative evaluation of enhancement patterns in focal solid liver lesions with Gd-EOB-DTPA-enhanced MRI. PloS one 2014; 9: e100315

4 Zech CJ, Korpraphong P, Huppertz A et al. Randomized multicentre trial of gadoxetic acid-enhanced MRI versus conventional MRI or CT in the staging of colorectal cancer liver metastases. The British journal of surgery 2014; 101: 613-621

5 Bioulac-Sage P, Balabaud C, Zucman-Rossi J. Subtype classification of hepatocellular adenoma. Digestive surgery 2010; 27: 39-45

6 ESGAR 2014 Book of Abstracts. Insights into imaging. 5: 2014: 453 500

7 Kinner S, Steinweg $V$, Maderwald S et al. Bile duct evaluation of potential living liver donors with Gd-EOB-DTPA enhanced MR cholangiography: Single-dose, double dose or half-dose contrast enhanced imaging. European journal of radiology 2014; 83: $763-767$

8 Holzapfel K, Eiber MJ, Fingerle AA et al. Detection, classification, and characterization of focal liver lesions: Value of diffusion-weighted MR imaging, gadoxetic acid-enhanced MR imaging and the combination of both methods. Abdom Imaging 2012; 37: 74-82

9 Hoffmann R, Rempp H, Schraml C et al. Diffusion-weighted imaging during MR-guided radiofrequency ablation of hepatic malignancies: analysis of immediate pre- and post-ablative diffusion characteristics. Acta radiologica 2014

10 Beiderwellen K, Gomez B, Buchbender C et al. Depiction and characterization of liver lesions in whole body [(1)(8)F]-FDG PET/MRI. European journal of radiology 2013; 82: e669-e675

11 Albrecht MH, Wichmann JL, Muller C et al. Assessment of colorectal liver metastases using MRI and CT: impact of observer experience on diagnostic performance and inter-observer reproducibility with histopathological correlation. European journal of radiology 2014; 83: $1752-1758$

12 Mitchell DG, Bruix J, Sherman M et al. LI-RADS (Liver Imaging Reporting and Data System): summary, discussion, and consensus of the LI-RADS Management Working Group and future directions. Hepatology 2015; 61: $1056-1065$

13 Schelhorn J, Theysohn J, Ertle J et al. Selective internal radiation therapy of hepatic tumours: is coiling of the gastroduodenal artery always beneficial? Clinical radiology 2014; 69: e216-e222

14 Theysohn JM, Ertle J, Muller S et al. Hepatic volume changes after lobar selective internal radiation therapy (SIRT) of hepatocellular carcinoma. Clinical radiology 2014; 69: $172-178$ 
15 Schelhorn J, Ertle J, Schlaak JF et al. Selective internal radiation therapy of hepatic tumors: procedural implications of a patent hepatic falciform artery. Springerplus 2014; 3: 595

16 Gibbs P, Gebski V, Van Buskirk M et al. Selective Internal Radiation Therapy (SIRT) with yttrium-90 resin microspheres plus standard systemic chemotherapy regimen of FOLFOX versus FOLFOX alone as first-line treatment of non-resectable liver metastases from colorectal cancer: the SIRFLOX study. BMC Cancer 2014; 14: 897

17 Ricke J, Bulla K, Kolligs $F$ et al. Safety and toxicity of radioembolization plus Sorafenib in advanced hepatocellular carcinoma: analysis of the European multicentre trial SORAMIC. Liver Int 2014

18 Theysohn JM, SchlaakJF, Muller S et al. Selective internal radiation therapy of hepatocellular carcinoma: potential hepatopulmonary shunt reduction after sorafenib administration. J Vasc Interv Radiol 2012; 23: 949-952

19 Seufferlein T, Porzner M, Becker T et al. S3-guideline exocrine pancreatic cancer. Zeitschrift fur Gastroenterologie 2013; 51: 1395-1440

20 Ariyama J, Suyama M, Ogawa $K$ et al. The detection and prognosis of small pancreatic carcinoma. International journal of pancreatology: official journal of the International Association of Pancreatology 1990; 7: 37-47

21 Yeo CJ, Cameron JL. Prognostic factors in ductal pancreatic cancer. Langenbeck's archives of surgery / Deutsche Gesellschaft fur Chirurgie 1998; 383: 129-133

22 Ishikawa O, Ohigashi H, Imaoka S et al. Minute carcinoma of the pancreas measuring $1 \mathrm{~cm}$ or less in diameter-collective review of Japanese case reports. Hepato-gastroenterology 1999; 46: 8-15

23 Krishna N, Tummala P, Reddy AV et al. Dilation of both pancreatic duct and the common bile duct on computed tomography and magnetic resonance imaging scans in patients with or without obstructive jaundice. Pancreas 2012; 41: 767-772

24 Al-Hawary MM, Francis IR, Chari ST et al. Pancreatic ductal adenocarcinoma radiology reporting template: consensus statement of the Society of Abdominal Radiology and the American Pancreatic Association. Radiology 2014; 270: 248-260

25 Gaujoux S, Brennan MF, Gonen $M$ et al. Cystic lesions of the pancreas: changes in the presentation and management of 1424 patients at a single institution over a 15-year time period. Journal of the American College of Surgeons 2011; 212: 590-600; discussion -3

26 Valsangkar NP, Morales-Oyarvide V, Thayer SP et al. 851 resected cystic tumors of the pancreas: a 33-year experience at the Massachusetts General Hospital. Surgery 2012; 152: S4-S12

27 Brambs HJ, Juchems M. Radiological evaluation of intraductal papillary mucinous neoplasm. Der Chirurg Zeitschrift fur alle Gebiete der operativen Medizen 2012; 83: 116-122

28 Suzuki Y, Atomi Y, Sugiyama M et al. Cystic neoplasm of the pancreas: a Japanese multiinstitutional study of intraductal papillary mucinous tumor and mucinous cystic tumor. Pancreas 2004; 28: 241 - 246

29 Grenacher L, Strauß A, Bergmann F et al. Cyst features and Risk of Malignancy in Intraductal Papillary Mucinous Neoplasms of the Pancreas: Imaging and Pathology. Zeitschrift Viszeralmedizin 2015: 1

30 Tanaka M, Fernandez-del Castillo C, Adsay V et al. International consensus guidelines 2012 for the management of IPMN and MCN of the pancreas. Pancreatology 2012; 12: 183-197
31 Kloppel G, Luttges J. WHO-classification 2000: exocrine pancreatic tumors. Verhandlungen der Deutschen Gesellschaft fur Pathologie 2001; 85: 219-228

32 Brambs HJ, Juchems MS. Diagnostik von Pankreastumoren. Radiologie up2date 2010; 10: 311 - 334

33 Neumann HP, Dinkel E, Brambs $H$ et al. Pancreatic lesions in the von Hippel-Lindau syndrome. Gastroenterology 1991; 101: 465-471

34 Brambs HJ, Juchems M. Radiologische Diagnostik zystischer Pankreastumoren. Viszeralmedizin 2011; 27: 205-213

35 Baek JH, Lee JM, Kim SH et al. Small (<or=3 cm) solid pseudopapillary tumors of the pancreas at multiphasic multidetector CT. Radiology 2010; 257: 97-106

$36 \mathrm{Yu}$ MH, Lee JY, Kim MA et al. MR imaging features of small solid pseudopapillary tumors: retrospective differentiation from other small solid pancreatic tumors. Am J Roentgenol American journal of roentgenology 2010; 195: 1324-1332

37 Klauss M, Stiller W, Fritz F et al. Computed tomography perfusion analysis of pancreatic carcinoma. J Comput Assist Tomogr 2012; 36: 237 242

38 Klauss M, Stiller W, Pahn G et al. Dual-energy perfusion-CT of pancreatic adenocarcinoma. European journal of radiology 2013; 82: 208 - 214

39 Skornitzke S, Fritz F, Klauss $M$ et al. Qualitative and quantitative evaluation of rigid and deformable motion correction algorithms using dual-energy CT images in view of application to CT perfusion measurements in abdominal organs affected by breathing motion. The British journal of radiology 2015; 88: 20140683

40 Bittencourt LK, Matos C, Coutinho AC et al. Diffusion-weighted magnetic resonance imaging in the upper abdomen: technical issues and clinical applications. Magn Reson Imaging Clin N Am 2011; 19: 111 - 131

41 Klauss M, Lemke A, Grunberg K et al. Intravoxel incoherent motion MRI for the differentiation between mass forming chronic pancreatitis and pancreatic carcinoma. Investigative radiology 2011; 46: 57-63

42 Barral M, Taouli B, Guiu B et al. Diffusion-weighted MR imaging of the pancreas: current status and recommendations. Radiology 2015; 274: $45-63$

43 Hoffmeister A, Mayerle J, Beglinger C. Chronic Pancreatitis German Society of D, Metabolic D. et al. S3-Consensus guidelines on definition, etiology, diagnosis and medical, endoscopic and surgical management of chronic pancreatitis German Society of Digestive and Metabolic Diseases (DGVS). Zeitschrift fur Gastroenterologie 2012; 50: 1176-1224

44 Schreyer AG, Jung M, Riemann JF et al. S3 guideline for chronic pancreatitis - diagnosis, classification and therapy for the radiologist. Fortschr Röntgenstr 2014; 186: $1002-1008$

45 Sarner M, Cotton PB. Classification of pancreatitis. Gut 1984; 25: 756 759

46 Yoshida K, Toki F, Takeuchi T et al. Chronic pancreatitis caused by an autoimmune abnormality. Proposal of the concept of autoimmune pancreatitis. Digestive diseases and sciences 1995; 40: 1561-1568

47 Rehnitz C, Klauss M, Singer $R$ et al. Morphologic patterns of autoimmune pancreatitis in CT and MRI. Pancreatology 2011; 11: 240-251

48 Rahib L, Smith BD, Aizenberg $R$ et al. Projecting cancer incidence and deaths to 2030: the unexpected burden of thyroid, liver, and pancreas cancers in the United States. Cancer research 2014; 74: 2913-2921

49 Strobel 0 , Hartwig W, Hackert T et al. Re-resection for isolated local recurrence of pancreatic cancer is feasible, safe, and associated with encouraging survival. Annals of surgical oncology 2013; 20: 964-972 